\title{
Relative Impact of Oral Rehabilitation on Quality of Life and Patient Satisfaction Following Mandibular Resection in a Sub-Urban Nigerian Tertiary Hospital
}

\author{
Obitade S. Obimakinde ${ }^{1}$, Adeyinka A. Adeleke ${ }^{2}$, Akinkunmi M. Akinpelu ${ }^{3}$, \\ Oluwaseun D. Abodunde ${ }^{4}$, Christopher O. Ibidun ${ }^{4}$
}

\begin{abstract}
${ }^{1}$ Senior Lecturer/Consultant, Oral and Maxillofacial Surgery, Ekiti State University Teaching Hospital, Ado-Ekiti, Nigeria ${ }^{2}$ Lecturer/Consultant Restorative Dental Surgeon, Federal Teaching Hospital, Ido-Ekiti, Formerly, Consultant, Restorative Dentistry, Ekiti State University Teaching Hospital, Ado-Ekiti, Nigeria

${ }^{3}$ Senior Registrar, Department of Restorative Dentistry, Obafemi Awolowo University Teaching Hospital, Ile-Ife, Formerly, Senior Dental Officer, Restorative Dentistry Unit, Ekiti State University Teaching Hospital, Ado-Ekiti, Nigeria

${ }^{4}$ Senior Dental Officer, Restorative Dentistry, Ekiti State University Teaching Hospital, Ado-Ekiti, Nigeria

Email: *oobimakinde@gmail.com, *obita de.obimakinde@eksu.edu.ng
\end{abstract}

How to cite this paper: Obimakinde, O.S., Adeleke, A.A., Akinpelu, A.M., Abodunde, O.D. and Ibidun, C.O. (2019) Relative Impact of Oral Rehabilitation on Quality of Life and Patient Satisfaction Following Mandibular Resection in a Sub-Urban Nigerian Tertiary Hospital. Open Journal of Stomatology, 9, 181-191.

https://doi.org/10.4236/ojst.2019.98019

Received: June 18, 2019

Accepted: August 26, 2019

Published: August 29, 2019

Copyright $\odot 2019$ by author(s) and Scientific Research Publishing Inc. This work is licensed under the Creative Commons Attribution International License (CC BY 4.0).

http://creativecommons.org/licenses/by/4.0/ (c) (i) Open Access

\begin{abstract}
Background: Resection of the mandible can lead to bony defect which has a direct bearing on the quality of life of the patient. Reconstruction of such defect is necessary to restore aesthetics but optimal functioning of the oral cavity can only be achieved with prosthodontic rehabilitation. We, hereby, report the impact of oral rehabilitation on patients' satisfaction and quality of life after mandibular reconstruction in our institution. Materials and Method: Patients who had oral rehabilitation following mandibular reconstruction from January 2010 to December 2015 were included. Patients' oral health related quality of life [OHRQL] before and after rehabilitation was evaluated using the head and neck module of the European Organization for Research and Treatment of Cancer [EORTC]. Oral functions and denture satisfaction were also evaluated with Visual Analogue Scale [VAS]. Data obtained were analyzed with SPSS version 20 and level of significance was set at $p<0.05$. Result: Of the 43 patients who had mandibular reconstruction during the study period, only 21 [48.8\%] had dental rehabilitation. Twelve patients [57.1\%] had conventional acrylic denture, 5 had fixed denture [bridge] while 4 patients had rehabilitation with dental implants. The mean follow-up period after rehabilitation was 8.42 months [Range: 6 - 22 months]. Although, OHRQL analysis revealed an improvement following rehabilitation, only the social aspect of the evaluation was statistically significant $[p<0.01]$. Also,
\end{abstract}


type of rehabilitation had a significant correlation with oral function as patients who received dental implant had better VAS score $[p=0.00]$. On the contrary, there was no statistically significant difference in aesthetics amongst the patients regardless of type of rehabilitation $[p=0.26]$. Conclusion: Findings from this study showed that there is improvement in OHRQL following dental rehabilitation. Also, Patients who were rehabilitated with dental implant had better oral function than those with fixed or conventional denture.

\section{Keywords}

Mandibular Reconstruction, Oral Rehabilitation, Implant, Denture, Oral Function

\section{Introduction}

Jaw resection secondary to malignant or benign tumors often leads to extensive composite defect which has a direct bearing on the quality of life of the patient [1] [2] [3]. Mastication, speech and aesthetics are often adversely affected [4]. Reconstruction of such defect is necessary to restore aesthetics but optimal functioning of the oral cavity is not achieved until a prosthodontic rehabilitation is carried out [2] [4] [5] [6]. The objectives of rehabilitation following a composite resection should include the restoration of the bony integrity of the mandible, soft-tissue replacement where necessary and prosthodontic treatment [7]. The goals of prosthodontic treatment include the provision of lip support, improving articulation and mastication, reducing drooling, and regaining favorable esthetics [4] [7].

Advances in microvascular surgery allow reconstruction level that is close to the pre-surgical anatomy of the mandible with good aesthetic outcome [1] [2] [4] [7]. However, restoration of masticatory functions following dental rehabilitation remains debatable in the literature [7] [8] [9]. The use of osseointegrated implant for dental rehabilitation is widely advocated because it restores oral function and aesthetics [2] [4] [7]. However, conventional dentures (either fixed or removable) have been shown to provide improved masticatory ability and restoration of quality of life following mandibular reconstruction [5] [8] [9]. Furthermore, conventional denture has been found to be useful in extensively irradiated site following malignant tumor excision [5] [9].

Reconstruction of mandibular defects with bone graft or composite flap provides a base for denture or implant for oral rehabilitation. Common donor sites for autogenic bone graft include the iliac crest, rib bone, tibia tuberosity and calvarium [10] [11]. However, the osteocutaneous fibula free flap appeared to be the reconstruction of choice for mandibular defect when free flap is desired [5] [6] [12]. Although the literature is replete with studies on reconstruction of segmental defect of the mandible, there is dearth of published work on the assessment of oral health related quality of life (OHRQL) after rehabilitation of the 
dentition, especially in Sub-Saharan Africa. This formed the basis for this study which aimed to assess the impact of oral rehabilitation on OHRQL and patients' satisfaction following mandibular reconstruction in our institution.

\section{Materials and Method}

The study population comprised all patients that had mandibular resection and immediate reconstruction with autogenic non vascularized bone graft (iliac crest or rib bone) at our institution between January 2010 and December 2015. Patients were referred for dental rehabilitation after a minimum period of 6 months following mandibular reconstruction. This was done to give some allowance for graft take, absence of disease recurrence and to allow patients ample time to decide whether or not to undergo prosthodontic treatment.

Data on patients' age, gender and type of lesion necessitating mandibular resection were documented on individual proforma. Mandibular defects were classified based on the criteria suggested by Urken et al. [12]. Patients who consented to participate in the study were followed up after completing dental rehabilitation and their OHRQL was assessed at 6 months after dental rehabilitation.

Assessment of OHRQL was done using the head and neck module of European Organization for Research and Treatment of Cancer [EORTC, H\&N35]. Permission to use this module was duly obtained from the EORTC study group (Appendix 1) this module contains items exploring side effects and symptoms of treatment. The score is measured on a $0-100$ scale and higher scores represent greater degree of problems. Each patient who had dental rehabilitation was made to complete the module six months post-rehabilitation. The impact of rehabilitation was determined by comparing the patient's score of the items on the module before and after rehabilitation. Similarly, denture satisfaction and oral functions were assessed using a 10 point visual analogue scale [VAS] with 0 being complete satisfaction and 10 least satisfaction.

The mean scores for each category were determined and statistical analysis done using SPSS version 20. Patients' demography was analyzed with descriptive statistics while the Wilcoxon matched-pairs signed-ranks test was used to compare the results on quality of life with the H\&N35 questionnaire pre and postrehabilitation. One-way Analysis of variance was used to compare the impact of rehabilitation type on patients' satisfaction regarding oral function and aesthetics. $P \leq 0.05$ was considered statistically significant.

\section{Result}

Forty-three patients had mandibular resection with reconstruction during the study period. Of these, only 21 subjects [48.8\%] successfully completed dental rehabilitation and follow up evaluation. There was a higher male preponderance $[\mathrm{M}: \mathrm{F}=1.7: 1]$ among the patients who had mandibular reconstruction while the converse is true for those who had rehabilitation $[\mathrm{M}: \mathrm{F}=0.9: 1]$. Similarly, the average age of those who had rehabilitation [24.72 \pm 4.02 years, range: $20-41$ 
years] was comparatively less than the overall age [28.91 \pm 9.43$]$ of all the patients [range: 20 - 61 years]. The age and sex distribution of the patients are shown in Table 1.

The commonest lesion necessitating jaw resection in this series was Ameloblastoma [67.4\%] followed by odontogenic fibromyxoma [9.3\%]. Chronic osteomyelitis [4.7\%] of the mandible was the least frequent lesion necessitating mandibular resection. Figure 1 shows the diagnosis of the lesions and frequency of rehabilitation. $61.9 \%$ of patients who had dental rehabilitation were earlier treated for Ameloblastoma. The location of mandibular defects according to Urken et al's [12] classification is shown in Table 2. Lateral defects of the mandible were found to constitute the majority among the patients who had mandibular reconstruction [41.8\%] and those who were subsequently rehabilitated [47.6\%] respectively.

All the 21 patients who had dental rehabilitation were successfully followed up on outpatient basis for a minimum of 6 months [mean: $8.42 \pm 3.21$ months, range: 6 - 22 months]. Twelve patients received conventional removable acrylic denture, 4 had osseointegrated dental implants while the remaining 5 patients had fixed partial denture [bridge]. The distribution of the mode of rehabilitation was shown in Figure 2.

All rehabilitated patients in this series completed the H\&N35 Quality of life assessment questionnaire and the mean scores for the relevant parameters were shown in Table 3. In all the variables tested with the questionnaire, all the patients attested to an overall improvement in OHRQL following dental rehabilitation. However, of all the parameters measured, only social integration showed a statistically significant improvement following dental rehabilitation $[P<0.01]$.

Patients' satisfaction following rehabilitation among the three groups was compared with VAS. Indicators of satisfaction measured with VAS include oral functions and aesthetic satisfaction. Oral function encompasses masticatory performance, deglutition and speech. The mean VAS score for the three groups was shown in Table 4. Of the four patients who had dental implants, one had osseointegrated implants replacing 34, 35, 36 and 37] while the other three patients had implant retained dentures covering longer area of the dental arch [31 36, 33 - 45 and 41 - 46 respectively]. This group of patients reported better

Table 1. Age and sex distribution of the patients.

\begin{tabular}{ccccc}
\hline Age (years)/Sex & \multicolumn{2}{c}{ Reconstructed patients } & \multicolumn{2}{c}{ Rehabilitated patients } \\
\hline $21-30$ & Male & Female & Male & Female \\
$31-40$ & 11 & 8 & 6 & 7 \\
$41-50$ & 8 & 6 & 3 & 3 \\
$>51$ & 4 & 2 & 1 & 1 \\
Total & 4 & 0 & 0 & 0 \\
\hline
\end{tabular}




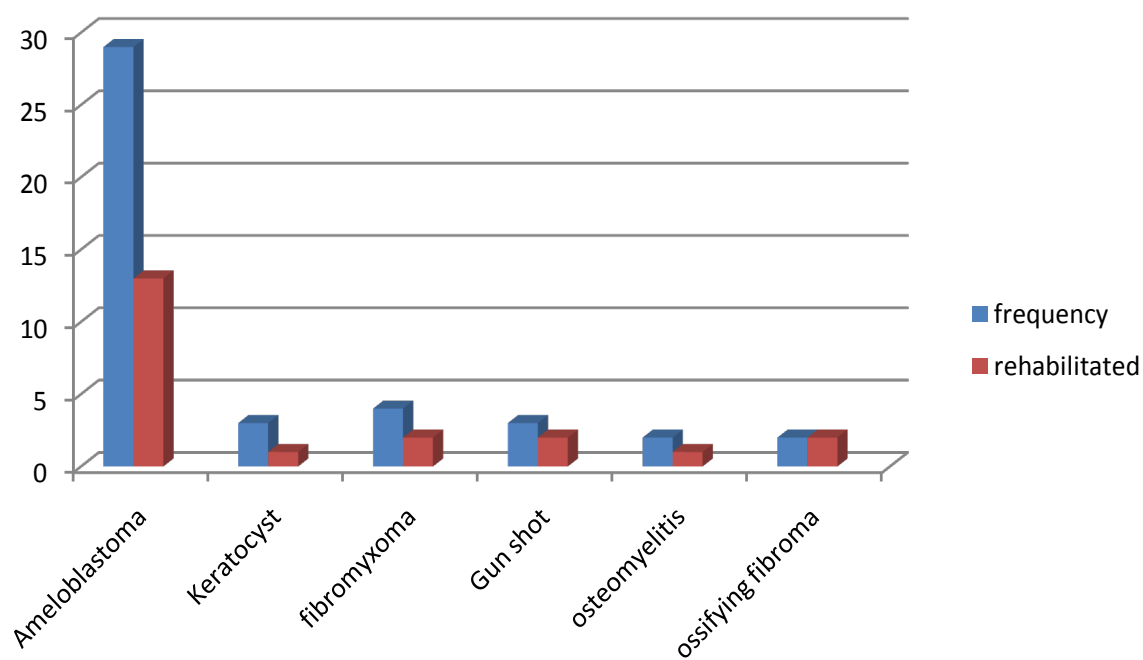

Figure 1. Mandibular lesion and the frequency of rehabilitation. Patients with Ameloblastoma constituted the majority of those who have resection [ $\mathrm{n}=29,67 \%]$ and Dental rehabilitation $[\mathrm{n}=13,61 \%$ ] respectively. Fibromyxoma, Gun shot and ossifying fibroma accounted for 2 patients each [9.5\%] among those who had Dental rehabilitation.

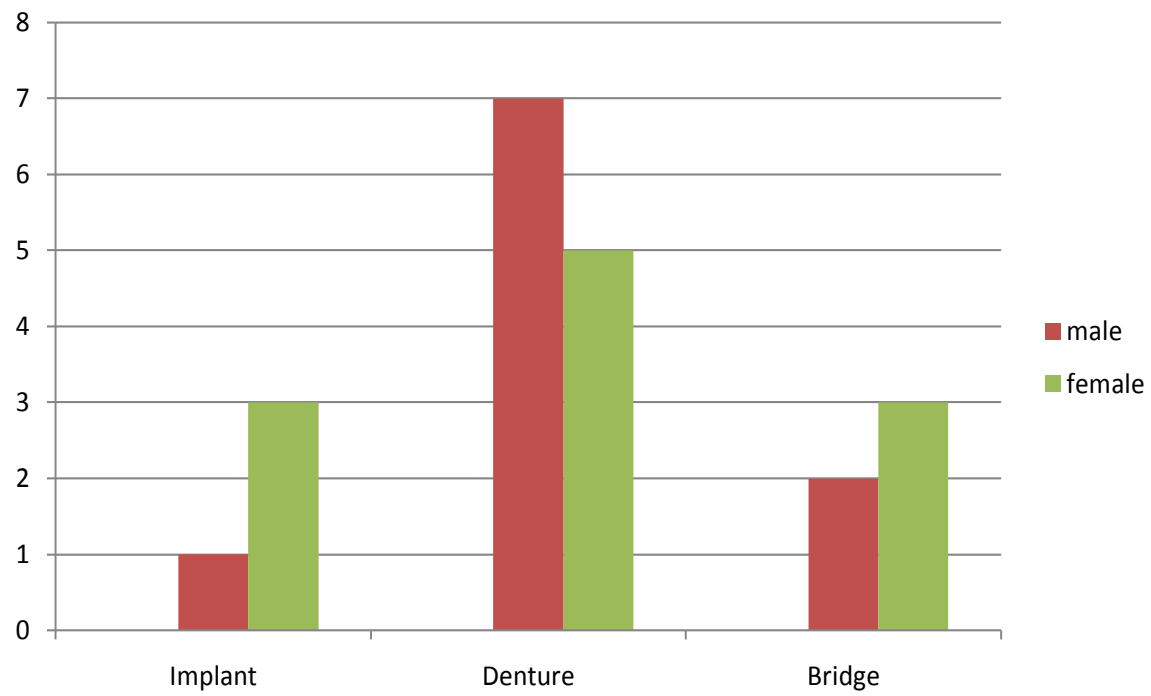

Figure 2. Mode of rehabilitation. A little over half of the rehabilitated patients had conventional Denture $[\mathrm{n}=12,57.1 \%]$ while the Implant and bridge groups had 4 [19.1\%] and 5 [23.8\%] patients respectively.

Table 2. Classification of Mandibular defects.

\begin{tabular}{ccc}
\hline Location of mandibular defect & reconstruction [n] & rehabilitation [n] \\
\hline Central & 4 & 4 \\
Lateral & 18 & 10 \\
Overlapping [central/lateral] & 9 & 7 \\
Lateral/posterior free end & 12 & 0 \\
Total & 43 & 21 \\
\hline
\end{tabular}


Table 3. Result of functional assessments and quality of life with the H\&N35 questionnaire.

\begin{tabular}{ccc}
\hline H\&N35 & Before rehabilitation & After rehabilitation \\
\hline Soreness in the mouth & T1 & T2 \\
Swallowing liquids & 55 & 42 \\
Swallowing solid food & 41 & 34 \\
Sense of taste & 51 & 46 \\
Chewing & 40 & 33 \\
Speech & 61 & 49 \\
Dry mouth & 44 & 39 \\
Appearance/Social integration & 40 & 38 \\
Talking & 64 & $23^{* *}$ \\
Mouth opening & 60 & 44 \\
Nutritional supplement/feeding tube & 46 & 37 \\
\hline
\end{tabular}

${ }^{* *} p<0.01$ [Wilcoxon matched-pairs signed-ranks test]; H\&N35 scale: from "0" to "100" ["0": negligible handicap, "100": maximum handicap].

Table 4. Mean VAS score of patients according to rehabilitation method.

\begin{tabular}{ccc}
\hline Rehabilitation/Mean VAS score & Aesthetics & Oral functions \\
\hline Denture & 3.5 & 6.2 \\
Implant & 2.9 & 2.5 \\
Fixed denture [bridge] & 3.0 & 5.0 \\
\hline
\end{tabular}

One way ANOVA: Oral function $\mathrm{F}=116.55$, Sig; $p=0.00$; Aesthetics $\mathrm{F}=1.397$, Sig; $p=0.26$.

mean score for oral functions which was statistically significant compared to those who had rehabilitation with conventional denture and bridge $[p=0.00]$. Also, those who had rehabilitation with fixed denture [bridge] had a statistically significant better mean score for oral functions than those who received only conventional denture [ $p=0.02]$. Regarding aesthetic satisfaction, all rehabilitated patients in the three groups had comparative mean VAS score, although the implant group had a better mean score, this was found not to be statistically significant $[p=0.26]$.

\section{Discussion}

The loss of a segment of jaw bone to tumor resection, infective process or trauma can have an adverse effect on the quality of life of affected individuals. Restoration of such defect is mandatory to improve appearance but optimal satisfaction is not achieved until full dental rehabilitation is carried out [2] [7]. It is evident from the literature that reconstruction with vascularized bone and subsequent rehabilitation with dental implants is the gold standard in the management of continuity defect of the jaws [3] [6] [13] [14] [15]. However, clinicians 
have equally reported appreciable outcome following rehabilitation with conventional denture, either removable or fixed, after mandibular reconstruction [5] [8] [9]. An assessment of OHRQL following jaw reconstruction and dental rehabilitation is therefore important in order to gauge the impact of treatments on patients' well-being.

Nearly half of the patients [48.8\%] who had mandibular resection in this study were successfully rehabilitated. This is higher than reports from similar studies where minor proportion of patients undergoes rehabilitation [2] [6] [8]. The difference may be due to the fact that patients with malignant disease were not part of this survey unlike the studies cited where such patients formed part of the cohort. The mean age of patients who had oral rehabilitation in this study was lower than those of Hendepool et al. [2] and Curtis et al. [7]. The mean age in those studies was higher because they incorporated older patients with mostly malignant lesions. Also, the mean age of the patients who had rehabilitation is comparatively lower than those who were not rehabilitated. We are of the opinion that age played a major role in determining whether patients will embrace rehabilitation or not. Also, more female patients had dental rehabilitation compared to males. Aesthetic and social inclination may play a major role as females tend to have better health care seeking attitude and are more aesthetically conscious than males.

Ameloblastoma was the most frequent benign lesion necessitating jaw resection in this study. This is similar to other studies where ameloblastoma was found to be the most frequent benign lesion in patients undergoing mandibular resection [9] [10]. However, some studies cited on dental rehabilitation and OHRQL involved malignant oral lesions majorly [2] [4] [6] [12]. Malignant oral lesions were not part of this series because nearly all cases are lost to follow up after referral for chemo-radiation treatment following surgery as we currently do not have services for radiation therapy in our young institution.

Researchers have employed various tools to assess OHRQL of rehabilitated patients following mandibular reconstruction. These include the Performance Status Scale for Head and Neck Cancer, Oral Health Impact Profile, The University of Washington Oral Health Quality of life Questionnaire and the European Organization for Research and Treatment of Cancer [EORTIC] Quality of Life Head and Neck Module [QLQ-H\&N35]. However, Quality-of-life assessment has increasingly moved towards a modular approach which allows assessments to be tailored to specific patient groups (through use of disease-specific modules), while preserving comparability across studies through use of the general measures [16]. Such an approach is embedded in the European Organization for Research and Treatment of Cancer (EORTC) Quality of Life Questionnaire which was employed for this study [16] [17].

Although, the mean scores on evaluation of OHRQL using the H\&N35 questionnaire revealed general improvement on all the parameters evaluated following dental rehabilitation, only the social aspects were statistically significant following comparative analysis $[p=0.01]$. This is in agreement with previous re- 
ports which suggested that dental rehabilitation has more impact on patients' cosmetic appearance than other aspects of oral functions [2] [4] [7] [18].

Some authors have examined masticatory performance as a measure of oral function following dental rehabilitation [7] [8] [9] [13] [18] [19] [20]. Methods employed include the use of bite force measurement, the use of test foods such as carrots and peanuts, assessment of tongue and cheek function, the use of Visual Analogue Scale to assess oral function and standardized questionnaires to get patients' level of satisfaction. We employed the Visual analogue scale in this study because it is convenient and patient can easily grade the level of performance of the prosthesis on the scale. Our findings which confirmed that the group with osseointegrated implant had a statistically significant better mean score for oral functions $[p=0.00]$ is in agreement with other similar studies where dental implants have been reported to have better impact on oral functions than conventional dentures [7] [8] [21] [22]. Also, the fact that patients who were rehabilitated with osseointegrated implant did not have a statistically significant mean VAS score for aesthetics agrees with findings of other authors of similar studies [2] [8] [9]. On the contrary, Curtis et al. [7] in their study reported that patients with osseointegrated implants have a statistically significant aesthetic satisfaction than those with conventional denture. However, a similar study by Komisar et al. [9] reported that prosthetic rehabilitation did not confer additional benefit to the patients following reconstruction.

One major limitation of this study was the relatively small sample size employed in comparative analysis, this is partly due to difficulty in getting patients to embrace rehabilitation following mandibular reconstruction. Economic reality also played a part as most subjects could not afford rehabilitation with osseointegrated implants. However, it is our belief that the data obtained from this study was sufficient enough for a pilot study on dental rehabilitation following mandibular reconstruction.

\section{Conclusion}

Although there is an improvement in patients' quality of life following oral rehabilitation, only the social aspect of OHRQL [smile and appearance] showed statistically significant improvement in this study. Furthermore, osseointegrated dental implants provided better improvement in oral functions than fixed or removable partial denture. We suggest a prospective study with larger sample size to overcome the limitations observed in this study.

\section{Conflicts of Interest}

The authors wish to declare that we do not have any conflict of interest regarding this study.

\section{References}

[1] Deleyiannis, F.W., Rogers, C., Lee, E., Russavage, J., Gastman, B., Dunklebarger, J., 
et al. (2006) Reconstruction of the Lateral Mandibulectomy Defect: Management Based on Prognosis and Location and Volume of Soft Tissue Resection. Laryngoscope, 116, 2071-2080. https://doi.org/10.1097/01.mlg.0000240858.88538.e1

[2] Hundepool, A.C., Dumans, A.G., Hofer, S.O.P., Fokkens, N.J.W., Rayat, S.S., Van der Meij, E.A. and Schepman, K.P. (2008) Rehabilitation after Mandibular Reconstruction with Fibula Free Flap: Clinical Outcome and Quality Life Assessment. International Journal of Oral and Maxillofacial Surgery, 37, 1009-1013.

https://www.sciencedirect.com/

https://doi.org/10.1016/j.ijom.2008.05.021

[3] Pappalardo, M., Tsao, C.K., Tsang, M.L., Zheng, J., Chang, Y.M. and Tsai, C.Y. (2018) Long-Term Outcome of Patients with or without Osseointegrated Implants after Resection of Mandibular Ameloblastoma and Reconstruction with Vascularized Bone Graft: Functional Assessment and Quality of Life. Journal of Plastic, Reconstructive \& Aesthetic Surgery, 71, 1076-1085.

https://doi.org/10.1016/j.bjps.2018.03.008

[4] Urken, M.L., Buchbinder, D., Weinberg, H., et al. (1991) Functional Evaluation Following Microvascular Oromandibular Reconstruction of the Oral Cancer Patient: A Comparative Study of Reconstructed and Nonreconstructed Patients. Laryngoscope, 101, 935-950. https://doi.org/10.1288/00005537-199109000-00004

[5] He, Y., Zhang, Z.Y., Zhu, H.G., Wu, Y.Q. and Fu, H.H. (2011) Double-Barrel Fibula Vascularized Flap with Dental Rehabilitation for Mandibular Reconstruction. Journal of Oral and Maxillofacial Surgery, 69, 2663-2669.

https://doi.org/10.1016/j.joms.2011.02.051

[6] Iizuka, T., Häfliger, J., Seto, I., Rahal, A., Mericske-Stern, R. and Smolka, K. (2005) Oral Rehabilitation after Mandibular Reconstruction Using an Osteocutaneous Fibula Free Flap with Endosseous Implants. Factors Affecting the Functional Outcome in Patients with Oral Cancer. Clinical Oral Implants Research, 16, 69-79. https://doi.org/10.1111/j.1600-0501.2004.01076.x

[7] Curtis, D.A., Plesh, O., Miller, A.J., et al. (1997) A Comparison of Masticatory Function in Patients with or without Reconstruction of the Mandible. Head \& Neck, 19, 287-296.

https://doi.org/10.1002/(SICI)1097-0347(199707)19:4<287::AID-HED7>3.0.CO;2-X

[8] Roumanas, E.D., Garrett, N., Blackwell, K.E., Freymiller, E., Abemayor, E., Wong, W.K., et al. (2006) Masticatory and Swallowing Threshold Performances with Conventional and Implant-Supported Prostheses after Mandibular Fibula Free-Flap Reconstruction. Journal of Prosthetic Dentistry, 96, 289-297.

https://doi.org/10.1016/j.prosdent.2006.08.015

[9] Komisar, A. (1990) The Functional Result of Mandibular Reconstruction. Laryngoscope, 100, 364-374. https://doi.org/10.1288/00005537-199004000-00007

[10] Okoje, V.N., Obimakinde, O.S., Arotiba, J.T., Fasola, A.O., Ogunlade, S.O. and Obiechina, A.E. (2012) Mandibular Defect Reconstruction with Non-Vascularized Iliac Crest Bone Graft. Nigerian Journal of Clinical Practice, 15, 224-227. https://doi.org/10.4103/1119-3077.97334

[11] Mehta, R.P. and Deschler, D.G. (2004) Mandibular Reconstruction in 2004: An Analysis of Different Techniques. Current Opinion in Otolaryngology \& Head and Neck Surgery, 12, 288-293. https://doi.org/10.1097/01.moo.0000131444.50445.9d

[12] Urken, M.L., Weinberg, H., Vickery, C., Buchbinder, D., Lawson, W. and Biller, H.F. (1991) Oromandibular Reconstruction Using Microvascular Composite Free Flaps: Report of 71 Cases and a New Classification Scheme for Bony, Soft Tissue, and Neurologic Defects. Archives of Otolaryngology_Head and Neck Surgery, 117, 
733-744. https://doi.org/10.1001/archotol.1991.01870190045010

[13] Bodard, A.G., Salino, S., Desoutter, A. and Deneuve, S. (2015) Assessment of Functional Improvement with Implant-Supported Prosthetic Rehabilitation after Mandibular Reconstruction with a Microvascular Free Fibula Flap: A Study of $25 \mathrm{~Pa}$ tients. Journal of Prosthetic Dentistry, 113, 140-145. https://doi.org/10.1016/j.prosdent.2014.08.005

[14] Fang, W., Liu, Y.P., Ma, Q., Liu, B.L. and Zhao, Y. (2015) Long-Term Results of Mandibular Reconstruction of Continuity Defects with Fibula Free Flap and Implant-Borne Dental Rehabilitation. The International Journal of Oral \& Maxillofacial Implants, 30, 169-178. https://doi.org/10.11607/jomi.3606

[15] Adell, R., Svensson, B. and Bågenholm, T. (2008) Dental Rehabilitation in 101 Primarily Reconstructed Jaws after Segmental Resections-Possibilities and Problems. An 18-Year Study. Journal of Cranio-Maxillofacial Surgery, 36, 395-402. https://doi.org/10.1016/j.jcms.2007.11.006

[16] Sherman, A.C., Simonton, S., Adams, D.C., Vural, E., Owens, B. and Hanna, E. (2000) Assessing Quality of Life in Patients with Head and Neck Cancer: Cross-Validation of the European Organization for Research and Treatment of Cancer (EORTC) Quality of Life Head and Neck Module (QLQ-H\&N35). Archives of Otolaryngology_Head and Neck Surgery, 126, 459-467.

https://doi.org/10.1001/archotol.126.4.459

[17] Bjordal, K., Hammerlid, E., Ahlner-Elmqvist, M., et al. (1999) Quality of Life in Head and Neck Cancer Patients: Validation of the European Organization for Research and Treatment of Cancer Quality of Life Questionnaire-H\&N35. Journal of Clinical Oncology, 17, 1008-1019. https://doi.org/10.1200/JCO.1999.17.3.1008

[18] Jacobsen, H.C., Wahnschaff, F., Trenkle, T., Sieg, P. and Hakim, S.G. (2016) Oral Rehabilitation with Dental Implants and Quality of Life Following Mandibular Reconstruction with Free Fibular Flap. Clinical Oral Investigations, 20, 187-192. https://doi.org/10.1007/s00784-015-1487-3

[19] Mochizuki, Y., Omura, K., Harada, H., Marukawa, E., Shimamoto, H. and Tomioka, H. (2014) Functional Outcomes with Dental Prosthesis Following Simultaneous Mandibulectomy and Mandibular Bone Reconstruction. Journal of Prosthodontic Research, 58, 259-266. https://doi.org/10.1016/j.jpor.2014.05.001

[20] Vijayaraghavan, N.V., Ramesh, G., Thareja, A. and Patil, S. (2015) Masticatory Efficiency after Rehabilitation of Acquired Maxillary and Mandibular Defects. Indian Journal of Dentistry, 6, 139-146. https://doi.org/10.4103/0975-962X.155880

[21] Matsui, Y., Neukam, F.W., Schmelzeisen, R. and Ohno, K. (1996) Masticatory Function of Postoperative Tumor Patients Rehabilitated with Osseointegrated Implants. Journal of Oral and Maxillofacial Surgery, 54, 441-447. https://doi.org/10.1016/S0278-2391(96)90117-2

[22] Gurlek, A., Miller, M.J., Jacob, R.F., Lively, J.A. and Schusterman, M.A. (1998) Functional Results of Dental Restoration with Osseointegrated Implants after Mandible Reconstruction. Plastic and Reconstructive Surgery, 101, 650. https://doi.org/10.1097/00006534-199803000-00011 


\title{
Your request for an EORTC-questionnaire Request ID : 59505
}

no-reply@eortc.be

to me -

\author{
Dear OBITADE OBIMAKINDE, \\ Thank you for registering on the EORTC Quality of Life Group website. \\ Your registration to obtain permission to use our tools has been approved. During the \\ registration process you agreed to our terms and conditions regarding the academic use \\ of our questionnaires. You can review the terms and conditions here. \\ Please find below the links to the requested tools: \\ Gastric Module (STO22) - Afrikaans \\ Head and Neck Module (H\&N35) - English
}

Appendix 1. Permission agreement to use the H\&N 35 Questionnaire by the EORTC study group. 